\title{
Studies on the Method of Size Reduction of Medicinal Compounds. IV.1) Solvate Formation of Chloramphenicol and Its Application to Size Reduction ${ }^{2}$
}

\author{
Indzo Himuro, ${ }^{3 a}$ ) Yoji Tsuda, ${ }^{3 b)}$ Keiji Sekiguchi, ${ }^{3 a}$ ) Isamu Horikoshi ${ }^{3 c}$ ) \\ and Мотоко KANKE ${ }^{3 a)}$ \\ School of Pharmaceutical Sciences, Kitasato University, ${ }^{\mathbf{3} a)}$ Research Laboratories, \\ Fujisawa Pharmaceutical Co., Ltd. ${ }^{3 b}$ ) and Faculty of Pharmaceutical \\ Sciences, Toyama University ${ }^{3}$ )
}

(Received February 9, 1971)

\begin{abstract}
Crystals of chloramphenicol obtained from about 30 kinds of solvents were analyzed by differential scanning calorimetry (DSC) for the detection of solvate formation. It was found that heterocyclic solvents such as pyridine and picolines formed solvates with chloramphenicol. Thermal behaviours of these solvates were further investigated by DSC under various conditions, by gas evolution analysis and by thermogravimetric analysis. The one-to-one solvate with pyridine was found most suitable to the size reduction by solvation and desolvation. The specific surface area of the desolvated chloramphenicol was $3.0-3.1 \mathrm{~m}^{2} / \mathrm{g}$. The heat of desolvation and the heat of transformation from the pyridinate to the desolvated chloramphenicol were $6.3 \pm 0.5 \mathrm{kcal} / \mathrm{mole}$ and $5.0 \pm 0.5 \mathrm{kcal} / \mathrm{mole}$, respectively. Also, the activation energy for the desolvation reaction was estimated by both the methods of Ozawa and Kissinger to be $20.6 \pm 1.6 \mathrm{kcal} / \mathrm{mole}$ and $20.0 \pm 0.8 \mathrm{kcal} / \mathrm{mole}$, respectively.
\end{abstract}

Previously, a method of size reduction was developed by desolvation from the solvates of organic compounds. ${ }^{4}$ Also, various conditions of the method were investigated for effective size reduction. 1) In the present study, solvate or molecular compound formation of chloramphenicol (CM) was examined by several thermal methods of analysis and from the results, an attempt was made to produce $\mathrm{CM}$ finer and more uniform in size than the one by the conventional mechanical process.

\section{Experimental}

Materials-1. CM: Commercial CM was recrystallized from aqueous ethanol. $\mathrm{mp}$ 150-151.5 . 2. Solvents: Reagent grade chemicals listed in Table I were purified by fractional distillation. If necessary, the solvents were dehydrated before distillation.

Crystal Appearance - - Habits of crystals deposited from various solvents differed considerably as shown in Table I. However, even when a solvate was formed between CM and a solvent, crystals of the solvate often took a similar shape as those of CM itself from other solvents. Also, the fact that the solvate crystals did not change opaque in a short time indicates that they were not easily desolvated by air-drying at room temperature. Therefore, solvate formation could not be recognized by simple observation of the crystals obtained.

Preparation of CM Pyridinate-1. Crystallization from Pyridine or Pyridine-Ethanol Mixture: $\mathrm{CM}$ was dissolved in hot pyridine or pyridine-ethanol mixture (1:1 by volume) and the solution was cooled at about $5^{\circ}$. Deposited crystals were immediately filtered and were air-dried. The pyridinate thus obtained turned pink on standing rather easily, especially when pure pyridine was used as a solvent.

2. Trituration with Pyridine: When CM was triturated in a mortar with a small amount of pyridine, the pyridinate was formed accompanying with a little heat of evolution.

1) Part III: K. Sekiguchi, I. Horikoshi and I. Himuro, Chem. Pharm. Bull. (Tokyo), 16, 2495 (1968).

2) Presented partly at the Kanto Local Meeting of the Pharmaceutical Society of Japan, Tokyo, June 1967.

3) Location: a) 5-9-1 Shirokane, Minatoku, Tokyo; b) 1 Kashimacho, Higashiyodogawaku, Osaka; c) 3190 Gofuku, Toyama.

4) K. Sekiguchi, K. Ito, E. Owada and K. Ueno, Chem. Pharm. Bull. (Tokyo), 12, 1192 (1964). 
TABLE I. Solvents used for Crystallization of Chloramphenicol and Forms of Crystals obtained from these Solvents

$\mathrm{H}_{2} \mathrm{O}, \mathrm{MeOH}$ (piled plates), EtOH (granular agglomerates), $n$-, and iso- $\mathrm{PrOH}$ (granular agglomerates), $n$ - $\mathrm{BuOH}$, Acetone (clustered needles), Methylethylketone, $\mathrm{CHCl}_{3}$ (plates, somewhat clustered), Dichloroethane (plates, not clustered), Trichloroethylene (needles, not clustered), Benzene and Toluene (plates, not clustered), Stylene (needles, not clustered), Chlorobenzene (needles, not clustered), p-Dioxane (clustered needles), Pyrol, Liquid ammonia, Ammonia water, mono-, di-, and tri-Butylamine, mono-, di-, and tri-Ethanolamine, Pyridine ${ }^{a}$ (clustered needles), $\alpha-,{ }^{a)} \beta-,{ }^{a)}$ and $\gamma$-Picoline, ${ }^{a}$ ) Quinoline, ${ }^{a)}$ Isoquinoline, ${ }^{a)}$ Piperidine, Piperazine, Morpholine ${ }^{a)}$

a) CM forms solvates with these solvents.

Weight Increase by Exposure to Pyridine or Quinoline Vapor-In a tight container of which temperature was maintained at $25 \pm 3^{\circ}$, weighing bottles containing powdered $\mathrm{CM}$ and a dish half filled with pyridine or quinoline were placed. After 5,20 and 48 hours, the absorbed amount of solvent was measured. As shown in Table II, absorption of pyridine was rapid, but the increase in weight after long exposure became much more than that calculated from the solvate composition and a solution was formed finally. On the other hand, CM absorbed only a negligible amount of quinoline vapor. Unlike the case of griseofulvin and $\mathrm{CHCl}_{3}{ }^{4,5)}$ preparation of solvates by vapor absorption was, therefore, not applicable to CM. Also, adsorption isotherm of pyridine vapor could not be measured.

TABLE II. Weight Increase of Chloramphenicol by Exposure to Pyridine and Quinoline Vapor ${ }^{a}$

\begin{tabular}{cccc}
\hline & $5 \mathrm{hr}$ & $20 \mathrm{hr}$ & $48 \mathrm{hr}$ \\
\hline Pyridine & $74 \%$ & $163 \%$ & partly liquefy \\
Quinoline & $0.4 \%$ & $1.0 \%$ & $1.6 \%$ \\
\hline
\end{tabular}

a) The amount of pyridine or quinoline contained in each solvate was taken as $100 \%$.

Differential Scanning Calorimetry (DSC) and Gas Evolution Analysis (GEA)_-A Perkin-Elmer DSC-1B Differential Scanning Calorimeter was used. The effluent gas was $\mathrm{N}_{2}$ for DSC and $\mathrm{He}$ for the simultaneous measurement of DSC and GEA. Thermal behaviors of the samples under semi-closed or open condition were measured in an ordinary aluminum pan with or without crimping. Also, analysis under closed condition was done using a liquid sample pan with crimping. In the former case, the sample weight was always $10.0 \mathrm{mg}$, while in the latter, smaller amount was used.

Thermogravimetric Analysis (TGA)_-TGA was done with a Perkin-Elmer TGS-1 Thermobalance. Samples weighing about $5 \mathrm{mg}$ were used, and a scan speed of $8-16^{\circ} / \mathrm{min}$ was employed.

Simultaneous Measurement of Differential Temperature and Dielectric Behavior-The apparatus used was the one previously reported by the authors. ${ }^{6)}$

Analysis with a Thermo-Microscope-Changes of the CM pyridinate crystals during heating were observed by Micrope Heating Stage Model 350 (Ernst Leitz Ltd.). With the same apparatus, photographs of the crystals before and after desolvation were taken and shown in Fig. 4 (A) and (B).

Particle Size Measurement by Sedimentation Method-Particle size and its distribution of the desolvated CM powder was measured by Micron Photo-Sizer Model MSKK (Toyodensoku Co.,Ltd.). Toluene was used as a suspending medium. A small amount of surfactant was added into the suspension to avoid flocculation as little as possible.

Measurement of Specific Surface Area_-The BET gas adsorption method was employed. The sample amount was $6-8.5 \mathrm{~g}$.

X-ray Diffraction Analysis_-The apparatus used was a Toshiba X-ray Diffraction Analyzer Type APX-102 (Ni filter; $\mathrm{Cu}-\mathrm{K} \alpha$ ray $\lambda=1.542 \AA$ ).

Elemental Analysis-1. Chloramphenicol Pyridinate: Anal. Calcd. for $\mathrm{C}_{11} \mathrm{H}_{12} \mathrm{O}_{5} \mathrm{~N}_{2} \mathrm{Cl}_{2} \cdot \mathrm{C}_{5} \mathrm{H}_{5} \mathrm{~N}: \mathrm{C}_{\text {, }}$ 47.78; H, 4.26; N, 10.45. Found: $\mathrm{C}, 47.93 ; \mathrm{H}, 4.26 ; \mathrm{N}, 10.51$. 2. Chloramphenicol Quinolinate: Anal. Calcd. for $\mathrm{C}_{11} \mathrm{H}_{12} \mathrm{O}_{5} \mathrm{~N}_{2} \mathrm{Cl}_{2} \cdot \mathrm{C}_{9} \mathrm{H}_{7} \mathrm{~N}: \mathrm{C}, 53.11 ; \mathrm{H}, 4.23 ; \mathrm{N}, 9.29$. Found: C, 53.29; H, 4.19; N. 9.20. 3. Chloramphenicol Morpholinate: Anal. Calcd. for $\mathrm{C}_{11} \mathrm{H}_{12} \mathrm{O}_{5} \mathrm{~N}_{2} \mathrm{Cl}_{2} \cdot \mathrm{C}_{4} \mathrm{H}_{9} \mathrm{ON}$ : C, 43.91; $\mathrm{H}, 5.16 ; \mathrm{N}, 10.24$. Found: C, $44.14 ; \mathrm{H}, 5.24 ;$ N. 10.37 .

5) K. Ueno, T. Yamaguchi, K. Yoshida, I. Hinata and K. Sekiguchi, Yakuzaigaku, 23, 284 (1963)

6) K. Sekiguchi, I. Horikoshi, I. Himuro and S. Mikami, Chem. Pharm. Bull. (Tokyo), 16, 1360 (1968). 


\section{Result and Discussion}

\section{Detection of Chloramphenicol Solvates and Their Thermal Behaviors}

To examine solvate formation, air-dried crystals obtained from various solvents (Table I) were applied to DSC both under semi-closed condition (with a crimped sample pan) and under open condition (without crimping), and to TGA. Among the solvents used, only heterocyclic ones such as pyridine, picolines and quinolines were found to react with $\mathrm{CM}$. The rest of the solvents did not form solvates and the DSC curves of the crystals deposited became the same as that of pure CM.

1) DSC Curves of Chloramphenicol Pyridinate-DSC curve of the pyridinate consists of three peaks [Fig. 1 (A)]. The first endothermic one is assigned to desolvation of pyridine, because the shape and the temperature of fall of this peak are much influenced by the experimental conditions, such as crimping and the rate of scanning. The small exothermic rise in the DSC curve is supposed to be due to crystallization of amorphous solid or the melt produced metastably. The second sharp endothermic peak represents melting of the desolvated CM. The fact that the peak maximum lies closely to the melting point of $\mathrm{CM}$ will indicate that even under semi-closed condition, nearly all the solvent in the pyridinate is removed from the system before the melting point is reached. The above assignment of the DSC peaks were also supported by the

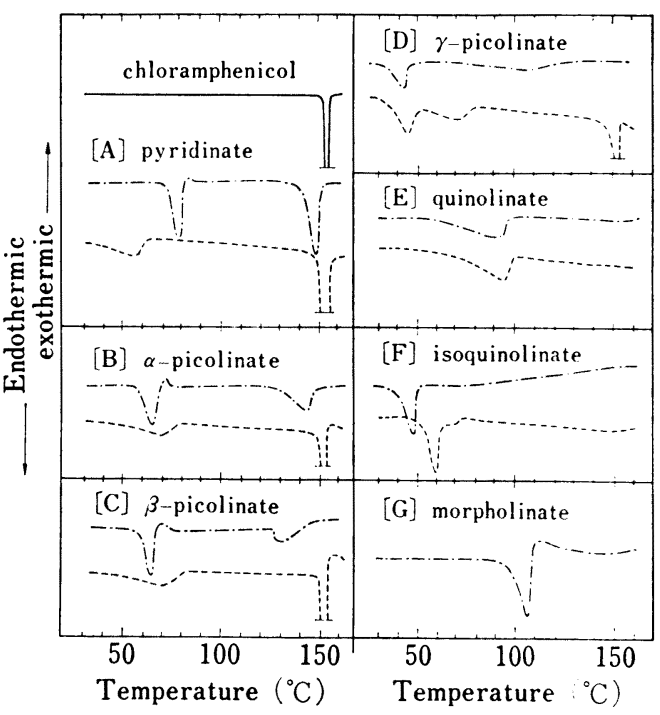

Fig. 1. DSC Curves of Various Solvates of Chloramphenicol

$$
\begin{aligned}
& \text { sample weight: } 10.0 \mathrm{mg} \\
& \text { scanning speed: } 8 \% \mathrm{~min} \\
& -.-1 \text { : semi-closed condition }
\end{aligned}
$$

results of simultaneous DSC and GEA, TGA and simultaneous measurement of differential temperature and dielectric behavior (Fig. 2 and 3).

2) DSC Curves of $\boldsymbol{\alpha}-, \boldsymbol{\beta}$-, and $\boldsymbol{\gamma}$-Picolinate_Each DSC curve exhibits two endothermic peaks [Fig. 1 (B), (C) and (D)]. However, the shapes and positions of these peaks under semiclosed condition differ considerably from peaks under open condition. The first broad peak of each uncrimped sample represents a composite heat effect due to evaporation from the solid solvate, peritectic fusion and subsequent evaporation from the melt, since the endothermic fall begins at relatively low temperature and a partial liquefaction was directly observed after the peak maximum. On the other hand, the corresponding peak of the crimped sample becomes sharper and is principally attributable to peritectic fusion.

Each of the second peaks in the DSC curves is ascribed to melting. Under open condition, as the solvent component evaporates completely until the end of the first peak, the second peak of each picolinate appears at the melting point of pure CM. Under semi-closed condition, however, the peak appears at a temperature lower than the melting point of CM because of the melting point depression due to solvent remained.

3) DSC Curves of Quinolinate and Isoquinolinate-These solvates exhibit only one endothermic peak irrespectively with or without crimping [Fig. 1 (E) and (F)]. The fact indicates that complete melting takes place in the temperature range of this peak. Therefore, it is supposed that the phase diagram of the system of CM and quinoline or isoquinoline will be the type that forms a compound with a congruent melting point. 
4) DSC Curve of Morpholinate - The DSC curve of the CM morpholinate consists of a large endothermic peak at about $90-110^{\circ}$ and an exothermic one at $110-130^{\circ}$ which is followed by a gradual endothermic fall [Fig. 1 (G)]. These heat effects will be explained similarly as those of picolinates.

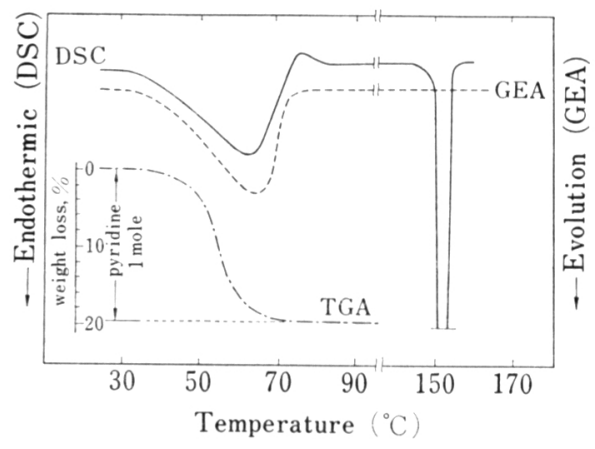

Fig. 2. DSC-GEA and TGA Curves of Chloramphenicol Pyridinate

$$
\begin{aligned}
& \text { scanning speed: } 2^{\circ} / \mathrm{min} \\
& \text { semi-closed condition } \\
& \text { heat of desolvation: } \Delta H_{d}=6.3 \pm 0.5 \mathrm{kcal} / \mathrm{mole}
\end{aligned}
$$

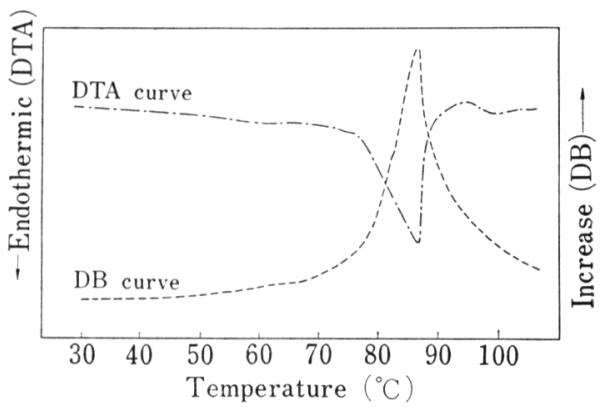

Fig. 3. Curves of Simultaneous Measurement of Differential Temperature and Dielectric Behavior of Chloramphenicol Pyridinate versus Temperature

$$
\begin{aligned}
& \text { sample weight: } 110.5 \mathrm{mg} \\
& \text { heating rate: } 1-2^{\circ} / \mathrm{min} \\
& \text { range: DTA } 25 \mathrm{mV}, \mathrm{DB} 5 \mathrm{mV}
\end{aligned}
$$

\section{Conditions for Desolvation of Chloramphenicol Pyridinate}

Based on the thermal behaviors of seven solvates, CM pyridinate was selected as the best one to apply to the solvation and desolvation method of size reduction. Unlike griseofulvin chloroformate, ${ }^{4}$ the pyridinate could not be prepared by contact with pyridine vapor; therefore, it was obtained by crystallization from pyridine or pyridine-ethanol mixture.

When a considerable amount of the pyridinate was heated directly at about $60^{\circ}$ in an open vessel, the desolvated CM crystals change color accompanying slight decomposition of CM. It is thought that this change would be due to free pyridine; therefore, to avoid color change, the process of eliminating pyridine was carried out by two steps, preliminary evacuation at room temperature for one hour to eliminate adhering pyridine and subsequent desolvation at $50-60^{\circ}$ under pressure of $30 \mathrm{mmHg}$. It is confirmed by DSC that elimination of pyridine will be over within 3 hours. The change of appearance of the pyridinate crystals after desolvation is shown in Fig. 4 (A) and (B). Though the desolvated particles assume the form of the original pyridinate crystal, they are easily divided into the primary particles by triturating lightly.

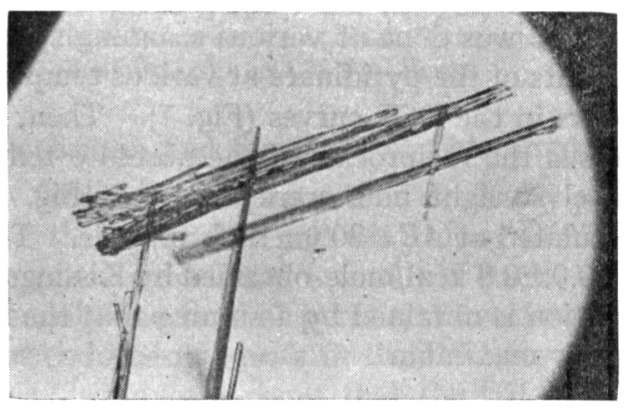

(A) before desolvation

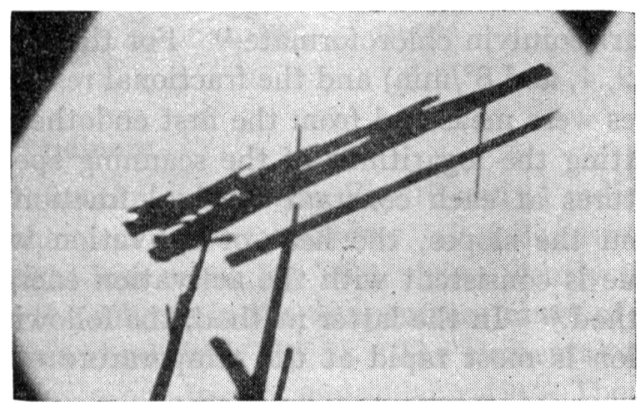

(B) after desolvation

Fig. 4. Chloramphenicol Pyridinate Crystals 


\section{Specific Surface Area of the Desolvated Chloramphenicol}

The specific surface area of the desolvated product was measured by BET method and was found to be $2.9-3.1 \mathrm{~m}^{2} / \mathrm{g}$. If the shape of the particles is assumed to be spherical, the mean particle diameter of $1.47 \mu$ is calculated by the following equation.

$$
\begin{aligned}
& d=K /(\rho \cdot s) \\
& d: \text { mean particle diameter } \\
& \rho: \text { density of particles (for CM, } \rho=1.48) \\
& s: \text { specific surface area measured } \\
& K: \text { shape factor (for sphere, } K=6)
\end{aligned}
$$

This data is highly consistent with the microphotograph of the particles in Fig. 5 (A) and (B). However, the particle size and its distribution (erroneous result was obtained by sedimentation method) were too large as compared with the above results. This is clearly attributed to agglomeration of the suspending particles in the liquid medium.

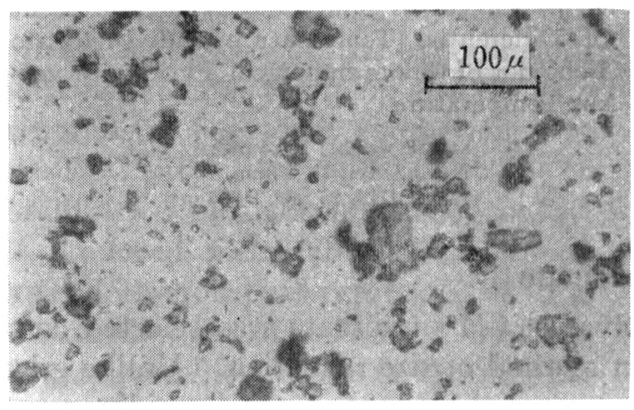

(A) by conventional method

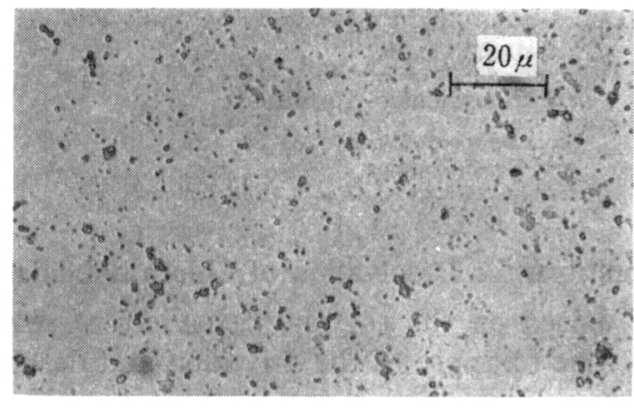

(B) by desolvation method

Fig. 5. Particles of Chloramphenicol

\section{Thermodynamic Constants of Chloramphenicol Pyridinate}

The heat of desolvation of CM pyridinate was measured by DSC under semi-closed condition. By substracting the area of the small exothermic peak from that of the first endothermic one, a calculated value of $\Delta H_{\mathrm{d}}=6.3 \pm 0.5 \mathrm{kcal} / \mathrm{mole}$ was obtained from 8 runs. The heat of transition from the pyridinate to the desolvate was also measured by conducting DSC under closed condition using a liquid sample pan. Since in this case no pyridine evaporate off from the system, the heat effect between 62 and $81^{\circ}$ in the DSC curve corresponds to the heat of transition (Fig. 6). Thus, the value was found to be $\Delta H_{\mathrm{t}}=5.0 \pm 0.5 \mathrm{kcal} / \mathrm{mole}$. To estimate the activation energy of desolvation, the method by Ozawa ${ }^{7)}$ was adopted, similarly as griseofulvin chloroformate. ${ }^{1)}$ For this purpose, DSC was done at various scanning speeds $\left(1,2,4\right.$, and $\left.8^{\circ} / \mathrm{min}\right)$ and the fractional residual amounts of the pyridinate at various temperatures were measured from the first endothermic peaks in the DSC curves (Fig. 7). Then, by plotting the logarithms of the scanning speeds versus the reciprocals of the absolute temperatures at each constant residual fraction, parallel straight lines were obtained (Fig. 8). From the slopes, the heat of activation was calculated at $\Delta E=20.6 \pm 1.6 \mathrm{kcal} / \mathrm{mole}$. This value is consistent with the activation energy of $20.0 \pm 0.8 \mathrm{kcal} / \mathrm{mole}$ obtained by Kissinger's method. ${ }^{8)}$ In the latter method, the following equation is obtained by assuming that the reaction is most rapid at the temperature of the peak maximum.

7) T. Ozawa, Bull. Chem. Soc. Japan, 38, 1881 (1965).

8) H.E. Kissinger, J. Res. Natl. Bur. Std., 57, 217 (1956); H. E. Kissinger, Anal. Chem., 29, 1702 (1957). 


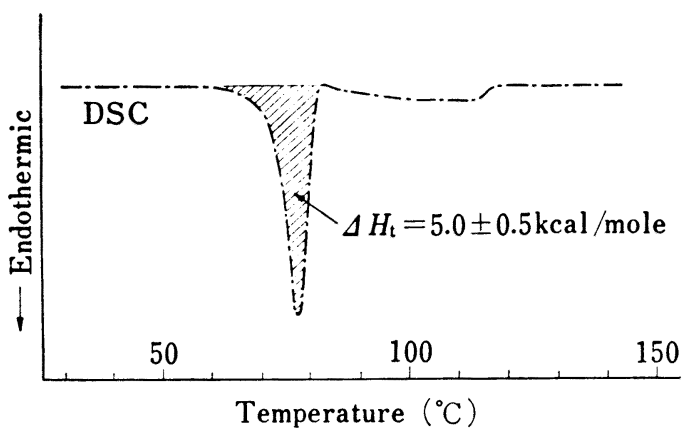

Fig. 6. DSC Curve of Chloramphenicol Pyridinate using Liquid Sample Pan (Closed Condition)

$$
\begin{aligned}
& \text { scanning speed: } 8 \% \mathrm{~min} \\
& \text { sample weight: } 2-4 \mathrm{mg}
\end{aligned}
$$

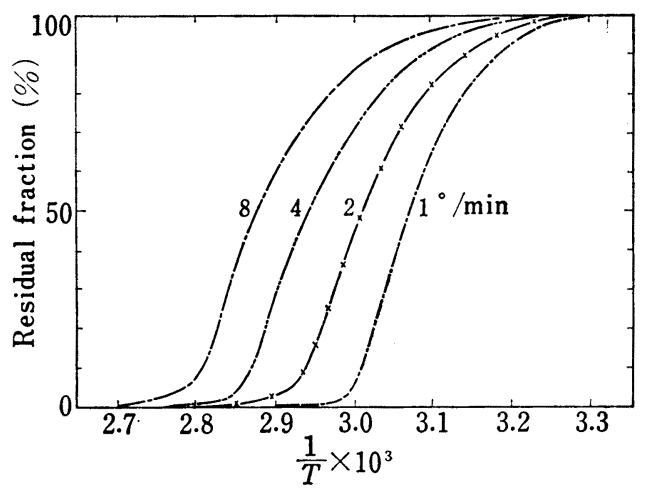

Fig. 7. The Cumulative DSC Curves of Desolvation of Chloramphenicol Pyridinate plotted against the Reciprocal of Absolute Temperature

$$
\begin{array}{ll}
\frac{d\left(\ln \phi T^{-2}\right)}{d\left(T^{-1}\right)}=-\frac{\Delta E}{R} & \\
\phi: \text { rate of heating }\left({ }^{\circ} \mathrm{C} / \mathrm{min}\right), & T: \text { maximum peak temperature }\left({ }^{\circ} \mathrm{K}\right) \\
\Delta E: \text { activation energy, } & R: \text { gas constant }
\end{array}
$$

As shown in Fig. 9, a linear relation is obtained between $\log \left(\phi T^{-2}\right)$ and $1 / T$, and from the slope, $\Delta E$ is calculated.

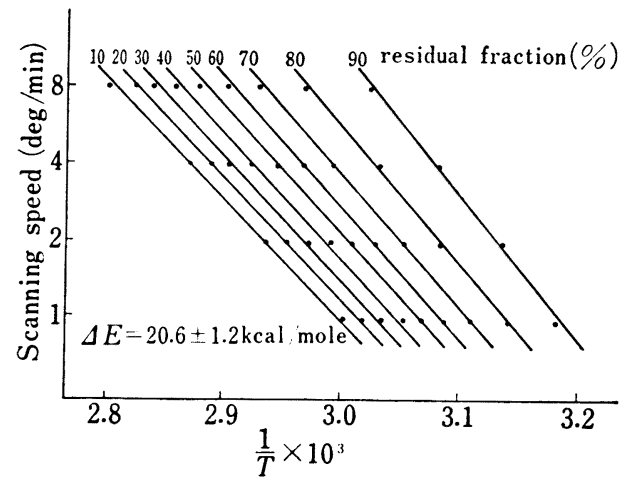

Fig. 8. Relationship between Logarithms of Scanning Speed and Reciprocal Absolute Temperature at Each Constant Residual Fraction of CM Pyridinate

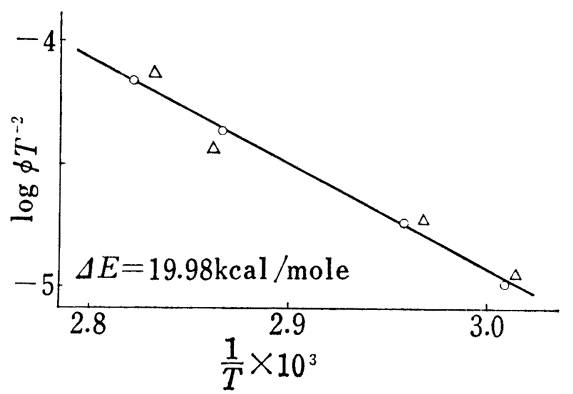

Fig. 9. Relationship between $\log \phi T^{-2}$ and $T^{-1}$ on Desolvation of Chloramphenicol Pyridinate

$$
\triangle: \text { DSC, } \quad \text { O: GEA }
$$

\section{Composition and Structure of Chloramphenicol Pyridinate}

The infrared spectra and the X-ray powder diffraction patterns in Fig. 10 and 11 indicate that the pyridinate and $\mathrm{CM}$ belong to different chemical species; however, after desolvation the pyridinate changes into CM itself. The weight-loss curve by TGA shows clearly that precisely one mole of pyridine dissociate off from the solvate in the temperature range of the first endothermic peak in the DSC curve under open condition (Fig. 2). The elemental analysis also gives a result that the CM pyridinate has a one-to-one molecular ratio. Though conclusive evidences as to the structure of the solvate is not available, it is supposed from the following facts that the bonding force involved is a hydrogen bond between the H-atom in 


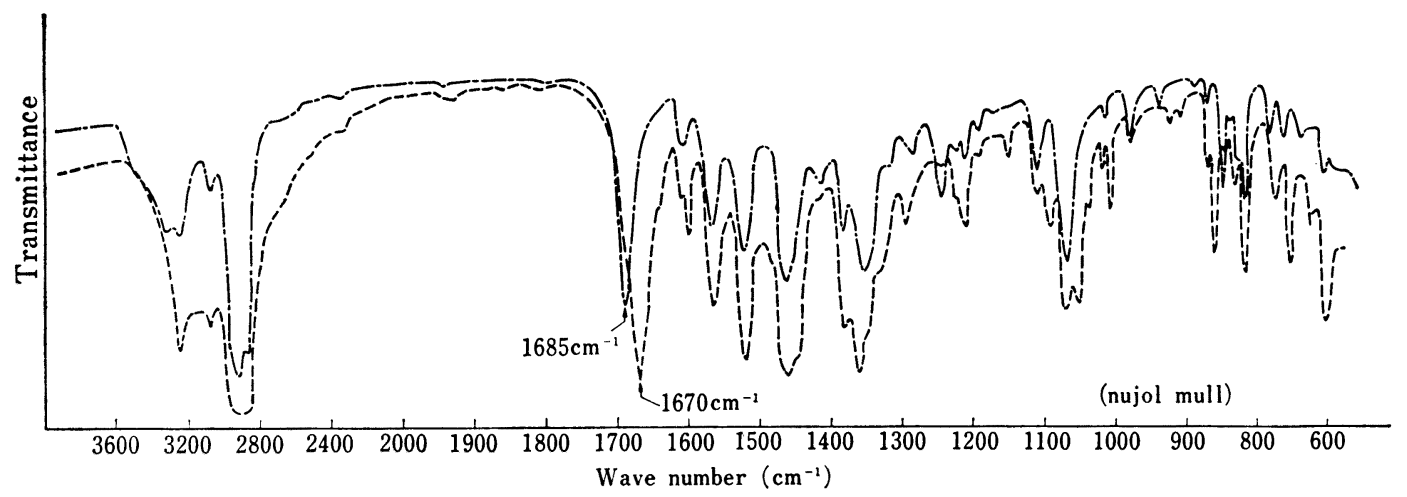

Fig. 10. Infrared Absorption Spectra of Chloramphenicol (-. - - ) and Chloramphenicol Pyridinate (----------)

the dichloroacetyl group of $\mathrm{CM}$ and the $\mathrm{N}$-atom of pyridine molecule. (1) The ultraviolet spectrum of the pyridinate solution was the same as a composite of those of CM and pyridine solutions. This suggests that the charge transfer force does not contribute to the bonding. (2) The heat of transition of the pyridinate is estimated at $5.0 \mathrm{kcal} / \mathrm{mole}$. The value is reasonable for the energy of hydrogen bonding. (3) In the infrared spectrum of pure CM (Fig. 10), the amide I band appears at $1685 \mathrm{~cm}^{-1}$; while the corresponding absorption of the pyridinate shifts to $1670 \mathrm{~cm}^{-1}$. This lowfrequency shift is probably attributed to the fact that the $\mathrm{C}-\mathrm{H}$ bond in the dichloroacetyl group has a proton-donating ability for hydrogen bonding by the electronic effect of $\mathrm{Cl}$ and $\mathrm{C}=\mathrm{O}$; however, once it is bonded with the $\mathrm{N}$-atom in pyridine, the double bond character of the amide $\mathrm{CO}$ group will be reduced. In this connection, bands due to the amide $\mathrm{NH}$ and nitrophenyl groups of both $\mathrm{CM}$ and the pyridinate appear in the same regions; this

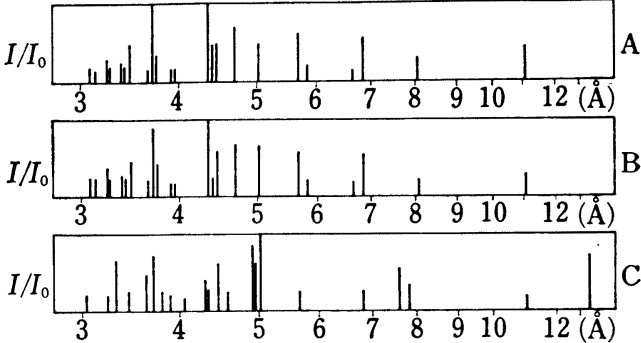

Fig. 11. X-ray Powder Diffraction Patterns

A: chloramphenicol

B : desolvated chloramphenicol C : chloramphenicol pyridinate suggests that these groups do not contribute to the solvate formation. On the other hand, the difference of absorption in $3600-3300$ and $1100-1000 \mathrm{~cm}^{-1}$ will probably be caused by the fact that inter- and intramolecular hydrogen bonding due to $\mathrm{OH}$ groups in the propandiol portion of $\mathrm{CM}$ is hindered by the pyridine molecule in the solvate.

\section{Conclusion}

As described in this paper, it is certain that the method by solvation and desolvation will be applied to the size reduction of CM. Since particles thus obtained are much finer and more uniform in size than those by the conventional mechanical pulverization, our method will be useful to prepare pharmaceutical products in which ultra fine powder of CM is needed, such as a sol-type parenteral suspension. However, $\mathrm{CM}$ pyridinate is thought to be less stable than griseofulvin chloroformate when the thermal behavior and other physical and chemical properties of the two solvates are compared; therefore, careful handling will be necessary for the $\mathrm{CM}$ pyridinate in the practical application.

Acknowledgement of Japan.
A part of this work was supported by the grant from the Ministry of Education 